\title{
Habilidades sociais em mulheres obesas: um estudo exploratório ${ }^{1}$
}

\author{
Sabrina Kerr Bullamah Correia ${ }^{2}$ \\ Zilda Aparecida Pereira Del Prette \\ Almir Del Prette
}

\begin{abstract}
Resumo
Dentre os aspectos psicológicos associados à obesidade, pode-se destacar a qualidade das relações interpessoais, supondo-se que as pessoas com sobrepeso podem, pelas restrições associadas, possuir déficits em habilidades sociais. Esta pesquisa avaliou, em uma amostra de 29 mulheres obesas mórbidas, seus principais déficits em habilidades sociais gerais e diante de demandas de alimentação; e a influência de características sociodemográficas e outros aspectos cognitivos e motivacionais sobre esses déficits. As mulheres responderam o Inventário de Habilidades Sociais, questões sobre Habilidades Assertivas na Alimentação (HAA) e um Questionário sobre Obesidade. A análise mostrou que as mulheres obesas apresentaram déficits principalmente em HAA, sendo semelhantes à amostra normativa nas habilidades gerais; não houve influência de características sociodemográficas; há aspectos cognitivos e motivacionais importantes a serem considerados na análise.

Palavras-Chave: Habilidades sociais; Assertividade; Autocontrole; Obesidade.
\end{abstract}

\section{Social skills in obese females: An exploratory study}

\begin{abstract}
Among the psychological aspects concerning obesity, the quality of the interpersonal relationships can be pointed out. The assumption is that the overweighted people, given their restrictions, may have deficits in social skills. This research aimed to verify, in a sample of 29 morbid obese women, their main deficits in general social skills and in those ones related to feeding; the influence of socio-demographic features and other cognitive or motivational aspects on those deficits. The women completed the Social Skills Inventory, questions about Assertive Skills associated with Feeding and a Questionnaire about Obesity. The results showed that obese women presented deficits mainly in Assertive Skills associated with Feeding, but they were similar to normative sample in the Social Skills Inventory; there were no influence of socio-demographic features; there are important cognitive and motivational aspects to be considered in this kind of study.
\end{abstract}

Keywords: Social skills; Assertiveness; Self-Control; Obesity.

A obesidade é uma doença e sua incidência vem aumentando consideravelmente em vários países, sendo considerada um problema de saúde pública pela Organização Mundial de Saúde. Embora não se disponha de dados mais recentes, o levantamento realizado no Brasil pelo Instituto Nacional de Alimentação e Nutrição (INAN, 1991) mostrou que, há cerca de 13 anos atrás, $32 \%$ da população adulta apresentava obesidade e sobrepeso, conforme avaliação do Índice de Massa Corpórea (IMC $\geq 25 \mathrm{~kg} / \mathrm{m}^{2}$ ), com maior prevalência entre as mulheres (38\%) do que entre homens (27\%). Os dados epidemiológicos fornecidos pela Associação Brasileira para o Estudo da Obesidade (ABESO, 1997), sobre inquérito realizado há cerca de sete anos atrás, nas regiões nordeste e sudeste (que representam dois terços da população brasileira) foram bastante semelhantes: $39 \%$ de mulheres e 38,5\% de homens. Destes, a proporção de obesos adultos (IMC $\geq 30 \mathrm{~kg} / \mathrm{m}^{2}$ ) foi de $7 \%$ entre homens e $12,4 \%$ entre mulheres, e a incidência de obesidade mórbida (IMC $\geq$ $40 \mathrm{~kg} / \mathrm{m}^{2}$ ) foi de $0,2 \%$ em homens, $0,8 \%$ em mulheres, com uma média de $0,5 \%$ de ambos os sexos.

$\mathrm{O}$ fato de a obesidade ter alcançado tais proporções está relacionado principalmente ao sedentarismo e ao excesso de calorias na alimentação. $\mathrm{Na}$ área de Psicologia existem poucos estudos sobre obesidade no Brasil, podendo-se apontar o grupo da USP-RP como referência em pesquisas sobre esse problema na infância (ver, por exemplo, Almeida, Nascimento, \& Quaioti, 2002; Del Prette, 2002).

A obesidade pode ser entendida como a deposição de gordura em excesso no organismo em razão da ingestão de maior quantidade de alimento do que a que pode ser utilizada para obtenção de energia (Guyton \&

${ }^{1}$ Este artigo é uma versão resumida e ligeiramente modificada da monografia de conclusão de curso da primeira autora, orientada pela segunda e reelaborada em co-autoria com o terceiro autor. Os autores agradecem a assessoria estatística do prof. dr. Lael Almeida de Oliveira neste trabalho.

2 Endereço para correspondência:

E-mail: sakbc@hotmail.com 
Hall, 1996; Leite, 1996). Um dos métodos utilizados para medir o acúmulo de gordura é o Índice de Massa Corpórea (IMC), que é calculado dividindo-se o peso (em quilos) pelo quadrado da altura (em metros) (Cotran, Kumar \& Collins, 2000). Os valores normativos são organizados em cinco classes, de acordo com o sexo (Halpern, 1998). Para o sexo feminino, as classes são definidas pelos seguintes valores do IMC: baixo (abaixo de 19); normal (de 19 a 23,9); obesidade leve (de 24 a 28,9); obesidade moderada (de 29 a 38,8); obesidade grave ou mórbida (acima de 39). Além de variáveis genéticas e biológicas, também estão envolvidos aspectos psicológicos, com alguns autores colocando-os como causas (Guyton \& Hall, 1996), outros como conseqüência (Friedman \& Brownell, 1995; Wadden \& Stunkard, 1985) e algumas pesquisas questionando a existência dessa relação (O’Neil \& Jarrel, 1992; Striegel-Moore \& Rodin, 1986).

Nesse sentido, alguns efeitos da obesidade podem ser decorrentes do preconceito e da discriminação contra pessoas obesas. Uma investigação conduzida por Molinari e Riva (1995) mostrou que mulheres obesas sentem-se indesejadas socialmente. Outras pesquisas também mostram que as pessoas obesas são vistas de forma mais negativa do que pessoas não obesas nos aspectos de inteligência e sucesso (Miller, Rothblum, Brand, Barbour \& Felício, 1990) e, portanto, socialmente evitadas ou até mesmo rejeitadas para certas funções de trabalho. Esses estereótipos preconceituosos e a discriminação contra pessoas com excesso de peso podem gerar problemas psicológicos e dificuldades variadas nas relações sociais que, por seu turno, podem complicar ainda mais o funcionamento adaptativo desse segmento da população.

Dentre os vários aspectos psicológicos potencialmente associados à obesidade, pode-se destacar a questão da qualidade das relações interpessoais. O estudo realizado por Miller e colaboradores (1990) mostrou que mulheres obesas causaram uma pior impressão nos demais, sendo consideradas menos agradáveis, menos habilidosas socialmente e com menor atratividade física que as não-obesas. Contudo, em um novo estudo, esses autores (Miller, Rothblum, Brand \& Felicio, 1995) encontram dados discrepantes do anterior: as mulheres obesas possuíam mais amigos, tinham mais apoio destes e se comportavam de forma mais competente em situações sociais do que não obesas.

Considerando a dificuldade de exercitar a recusa em geral (Del Prette \& Del Prette, 2003) e de recusar alimentos, em particular, uma hipótese pertinente seria que as pessoas obesas poderiam apresentar déficits de habilidades assertivas, entendendo-se estas como habilidade de enfrentamento de situações adversas, defesa de direitos, expressão de sentimentos negativos e discordância (Del Prette \& Del Prette, 2001). Klesges (1984) buscou verificar se isso realmente acontecia em todos os aspectos da vida do indivíduo (medidas globais da personalidade) ou se era restrito às questões referentes à alimentação (medidas específicas) em indivíduos. O autor verificou não haver diferenças entre pessoas obesas e não obesas quanto aos níveis globais da personalidade mas, nas medidas específicas, constatou que indivíduos obesos se avaliaram como menos assertivos em demandas relacionadas à alimentação ou dieta e mais depressivos em relação ao peso do que os indivíduos com pesos normais.

Os participantes do estudo de Klesges eram pessoas que não tinham experiência com qualquer tratamento contra a obesidade, podendo-se inferir que, para essas pessoas, a obesidade provavelmente não era incômoda. Os resultados, por não incluírem pessoas obesas que se incomodam com sua condição e buscam tratamentos na solução do problema, não são suficientes para qualquer explicação sobre obesidade e ausência de assertividade alimentar. O estudo de Klesges (1984) suscita, portanto, algumas questões para pesquisa: seus resultados se manteriam semelhantes em amostras de obesos sob tratamento, ou seja, pessoas que consideram a obesidade um problema? Seriam também essas pessoas socialmente mais habilidosas em termos gerais e menos habilidosas no que se refere à "assertividade alimentar"? Estudos enfocando essa questão podem ser particularmente importantes para indicar alternativas de intervenção relevantes na melhoria da qualidade de vida dessa clientela (por exemplo, o Treinamento de Habilidades Sociais); indicar fatores adicionais a serem levados em conta nos programas de controle da obesidade; verificar se tratamentos relacionados ao treinamento de habilidades sociais auxiliam também na perda de peso.

No Brasil, há ainda poucos estudos nacionais que buscam verificar possíveis relações entre habilidades sociais e obesidade e, em particular, com pessoas sob tratamento. Com base nas considerações anteriores, e tendo em vista a maior incidência feminina de obesidade em nosso meio, o objetivo dessa pesquisa foi analisar, em um grupo de mulheres obesas mórbidas sob tratamento médico: a) a incidência de déficits no repertório de Habilidades Sociais Gerais (HSG) e em subclasses de Habilidades Assertivas Alimentares (HAA); b) a relação entre esses conjuntos de habilidades; c) a relação dessas habilidades com características sociodemográficas e outros aspectos cognitivos e motivacionais do controle de peso.

\section{Método}

\section{Participantes}

Participaram deste estudo 29 mulheres obesas, pacientes do Hospital das Clínicas de Ribeirão Preto que estavam se submetendo a um tratamento médico para a obesidade sob orientação de um médico residente na área de nutrologia e um nutricionista. O critério de inclusão na 
amostra foi o sexo (feminino), grau de obesidade (obesas mórbidas, ou seja, IMC $>39$ ). A média do IMC no grupo estudado foi de 49,99; somente $27,6 \%$ não apresentavam outro problema de saúde além da obesidade e $75,9 \%$ tinham participado de tratamento anterior contra a obesidade.

Com relação às demais características, a idade variou de 21 a 58 anos (média $=37,57$; desvio padrão = 10,27); o maior contingente era de casadas (48,3\%), sendo as demais solteiras $(27,6 \%)$ ou divorciadas e viúvas $(6,9 \%)$, ou amasiadas (10,3\%); quanto à escolaridade, a maioria $(82,8 \%)$ tinha no máximo o primeiro grau completo; das demais, $6,9 \%$ obtiveram o segundo grau completo, outras $6,9 \%$ obtiveram o segundo grau completo e 3,4\% não tinham escolaridade. Quanto à ocupação, 48,3\% das mulheres trabalhavam fora de casa e $51,7 \%$, apenas nas atividades do lar.

\section{Instrumento}

Inventário de Habilidades Sociais - IHS-Del Prette (Del Prette \& Del Prette, 2001). Trata-se de um conjunto de 21 itens que descrevem situações interpessoais com demandas para o exercício de habilidades sociais. Em cada item, o respondente é solicitado a indicar a freqüência (em uma escala que varia de nunca/raramente a sempre/quase sempre) com que reage da forma indicada e os resultados são posteriormente transformados em um escore geral para a escala total e em escores parciais para as subescalas ou fatores que agrupam os itens em cinco fatores: F1 = Enfrentamento e auto-afirmação com risco; F2 = Auto-afirmação na expressão de sentimentos positivos; F3 = Conversação e desenvoltura social; F4 = Autoexposição a desconhecidos e situações novas; F5 = Autocontrole da agressividade e da raiva. Esse instrumento vem apresentando boas qualidades psicométricas (por exemplo, ver Bandeira e col. 2000; Del Prette, Del Prette \& Barreto, 1998) e tem parecer favorável do Conselho Federal de Psicologia (Edital CFP no 1 de 17/7/2003).

Questöes sobre Habilidades Assertivas Alimentares (HAA). Referem-se a seis itens adaptados de Klesges (1984), redigidos no padrão do IHS-Del Prette e incluídos aleatoriamente entre os itens deste instrumento: Quando me oferecem um lanche fora de hora, não aceito; Em uma saída com amigos para, por exemplo, comer pizza, consigo ficar sem comer resistindo à insistência dos meus amigos; Recuso-me a experimentar um novo produto de comida que está sendo oferecido no supermercado e parece gostoso; Recuso a oferta de repetir o prato nas refeiçōes; Quando alguém me oferece um pedaço de algo que está comendo (por exemplo: um pedaço de chocolate, uma mordida do lanche), eu recuso; Recuso a oferta de sobremesa. O conjunto desses itens produziu um alfa de Cronbach de 0,84, indicativo da sua validade.

Questionário de auto-relato da obesidade (QO). Foi elaborado de acordo com a literatura da área e envolveu questões abertas e fechadas sobre a obesidade e seus aspectos psicológicos, tais como avaliação do próprio comportamento de comer, motivação para emagrecer, expectativa quanto ao peso desejado e formas anteriores de tratamento.

\section{Procedimentos de coleta de dados}

O peso dos participantes foi obtido inicialmente por meio de consulta aos prontuários e os demais dados foram coletados em uma sala do Ambulatório do Hospital das Clínicas de Ribeirão Preto, no intervalo das consultas com o médico e nutricionista ou após essas consultas. Apenas seis participantes responderam ao questionário e ao inventário sozinhas; as demais pediram que a aplicadora lesse os enunciados e anotasse as respostas dadas. No prontuário constavam, além de outras informações, a altura e o peso do dia das participantes.

\section{Tratamento dos dados}

Os dados do IHS-Del Prette e das HAA foram, inicialmente, organizados em planilhas, efetuando-se a tabulação dos escores gerais e fatoriais e dos valores médios de cada item para a amostra como um todo. Com base nos escores, foi realizada a análise estatística descritiva (média, desvio padrão e mediana) e a inferencial (Teste-t e ANOVA) para a comparação entre esses valores e para a avaliação dos efeitos de características da amostra (escolaridade, estado civil, presença ou não de outro problema de saúde além da obesidade, realização ou não de tratamentos prévios da obesidade e trabalho fora de casa) sobre eles.

Além disso, os resultados foram comparados com os dados normativos preliminares da subamostra feminina de estudantes universitárias de 17 a 25 anos (Del Prette \& Del Prette, 2001; Del Prette, Del Prette \& Barreto, 1998), entendendo-se que essa alternativa era possível, com as devidas reservadas, considerando-se a falta de outros parâmetros em nosso meio.

Os resultados do QO, referentes ao IMC e IMC desejado, foram computados em médias do grupo, efetuando-se as comparações entre os dois índices. As respostas às questões abertas de relato foram organizadas nas classes e subclasses a seguir: Motivação para emagrecer: saúde; estética ("ficar mais atraente"; "ficar mais bonita"); aceitação social ("para evitar discriminação"; "para evitar chacotas"); trabalho ("para trabalhar melhor"; "porque quero trabalhar"; "por causa do emprego"). Situacões em que come mais freqüentemente e/ou em maior quantidade: ansiedade; tristeza; medo; frustração; problemas; discussão e raiva de alguém.

Realizou-se, ainda, uma análise de classificação e regressão por árvores tomando-se como variáveis dependentes as médias das IHS-Del Prette e HAA (técnica CART para o Answer Tree do Programa Estatístico SPSS, de Breiman, Friedman, Olshen \& Stone, 1984) e como parâmetros do ajuste de regressão: no pai $=10$, no filho $=5$, impureza mínima $=0,0001$. 


\section{Resultados}

O escore obtido pela amostra nos itens do IHSDel Prette foi de 92,1, o que corresponde à média da amostra de referência $(92,41)$, embora com variabilidade bem mais alta (desvio padrão de 23,74, contra o normativo de 15,49). Em comparação com as estudantes universitárias, pode-se dizer que a maioria das respondentes do presente estudo não possui repertório de habilidades sociais gerais deficitário, pelo menos quando se considera o conjunto total das habilidades avaliadas. $O$ valor médio por item, nas habilidades avaliadas pelo IHS-Del Prette $(2,32)$ pode ser comparado ao valor médio das HAA $(1,85)$, o que denota uma marcante diferença, ilustrada na Figura 1.

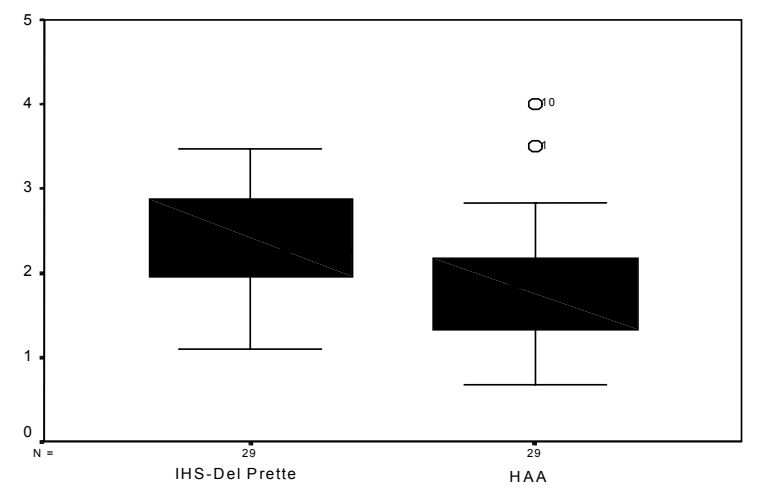

Figura 1 - Comparação entre as médias nos itens do IHSDel Prette e nos itens HAA
A análise estatística da diferença entre esses dois conjuntos de habilidades $(t=-2,363 ; \quad \mathrm{DP}=28$; $p=0,025)$ mostrou escores menores de freqüência (e, portanto, possivelmente, maior dificuldade) na emissão das HAA em relação às habilidades medidas pelo IHSDel Prette. A análise de correlação entre esses dois conjuntos de dados revelou-se não significativa $(\chi 2=$ $0,027$ e $p=0,889)$, ou seja, não houve uma tendência (direta ou inversa) de variação concomitante entre os dois indicadores. Em outras palavras, embora a média do escore de habilidades sociais tenha sido maior, isso não significou que os mesmos sujeitos com maiores escores neste indicativo apresentaram menores escores de habilidades assertivas alimentares ou vice-versa.

A constatação de que as mulheres obesas apresentam déficits nas HAA foi também confirmada na regressão por árvore. Tomando como variável dependente o escore médio no IHS-Del Prette, não foi possível construir uma árvore que indicasse variáveis preditoras para essa habilidade. No entanto, tomando como variável dependente a média das HAA, foi possível construir o diagrama de árvore que aparece na Figura 2.

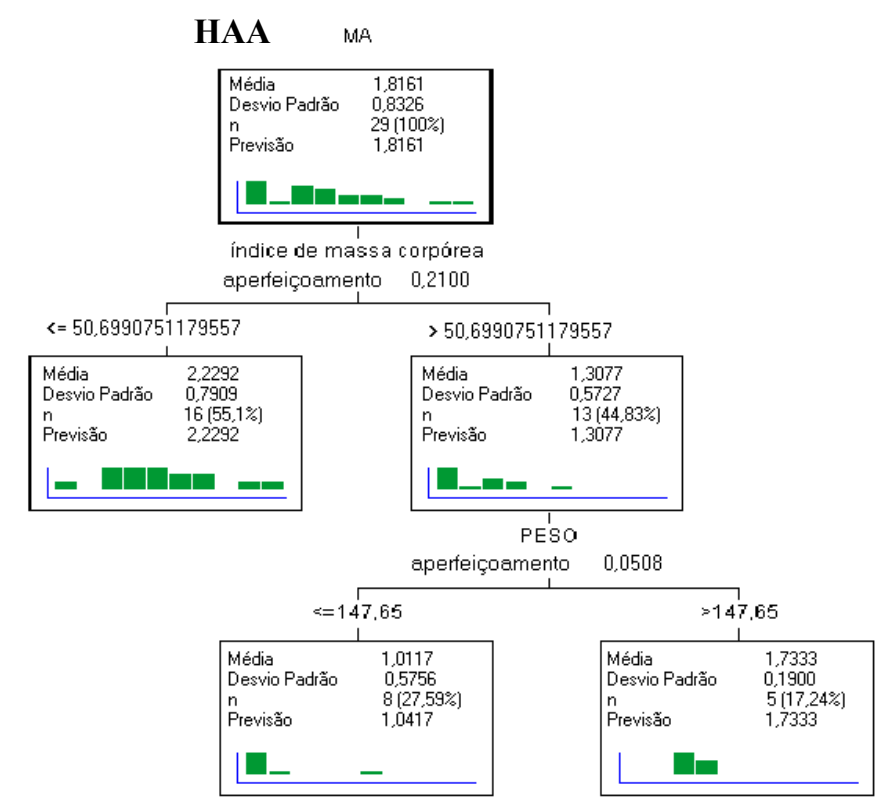

Figura 2 - Diagrama de árvore tendo como variável dependente HAA 
Conforme se vê na Figura 2, as 16 mulheres com IMC menor ou igual a 50,69 têm o escore médio de HAA de 2,229 e diferem significativamente das $13 \mathrm{mu}-$ lheres com IMC maior que 50,69, cujo escore de HAA é 1,307. Assim, verificou-se que as mulheres mais gordas (maior IMC) apresentaram, em média, escores mais baixos nas HAA e as demais foram um pouco melhor nessas habilidades. Embora nos dois grupos as médias obtidas nas HAA fossem baixas (apenas um pouco acima do ponto médio da escala), os dados evidenciam que, mesmo nessa faixa de variação, quanto mais obesa, maior é a dificuldade diante de demandas de assertividade ligadas a situações que envolvem alimentação (diferença entre os grupos de quase um ponto).
Entre as mulheres mais obesas (IMC maior que $50,69)$ houve ainda uma partição mostrando que aquelas com peso acima de 147 quilos apresentaram média maior nas HAA que aquelas com peso menor ou igual a 147. Esse dado é intrigante, pois sugere um efeito adicional do sobrepeso entre as respondentes de menor média em HAA: as "menos obesas" sendo ligeiramente menos assertivas que as "mais obesas", embora ambas com médias bastante baixas (abaixo do ponto 2 da escala), ou seja, numa variação que vai apenas de nunca (0) a quase nunca (1) e a às vezes (2).

Tomando-se as subescalas ou fatores do IHSDel Prette, efetuou-se uma comparação entre a amostra de obesas e as universitárias da amostra normativa. Esses dados são ilustrados na Figura 3.
F1- Enfrentamento e auto-afirmação com risco

F2- Auto-afirmação na expressão de sentimento positivo

F3- Conversação e desenvoltura social

F4- Auto-exposição a desconhecidos

F5- Autocontrole da agressividade e raiva

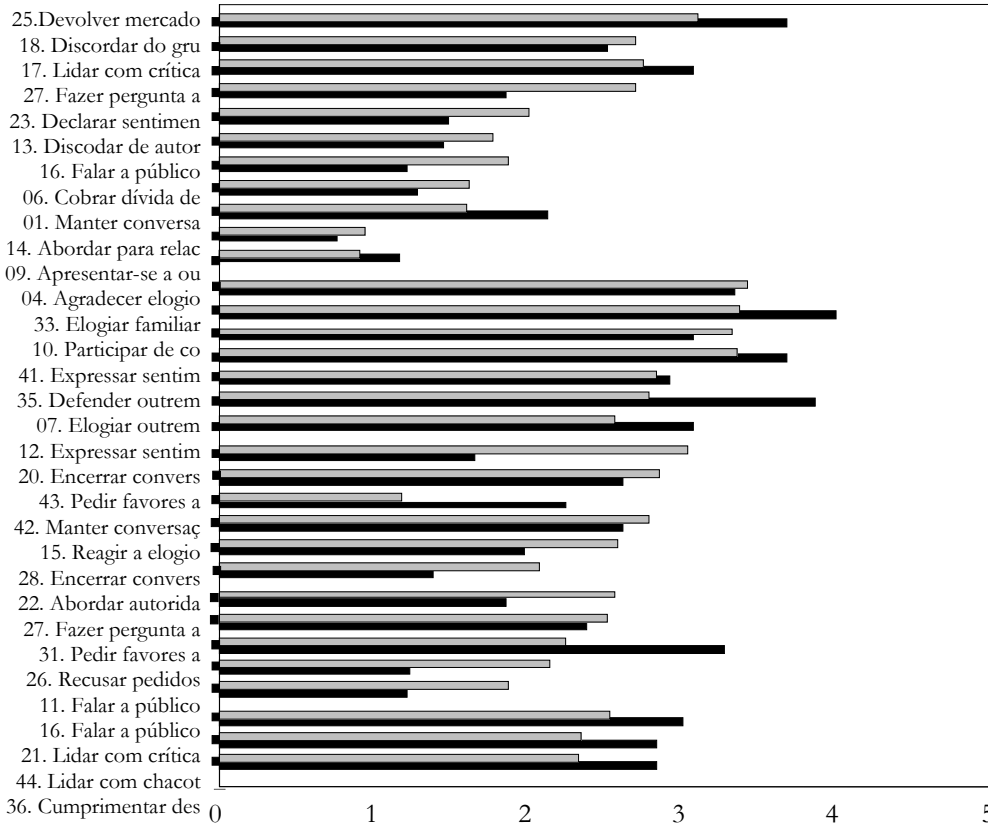

Amostra normativa

Amostra de obesas

Figura 3 - Distribuição dos valores médios das respondentes obesas nos itens do IHS-Del Prette em relação aos resultados das respondentes da amostra normativa

De acordo com a Figura 3, pode-se verificar que as mulheres obesas apresentam valores médios nominalmente mais elevados que os da amostra normativa, nos subconjuntos F2 e F5 (Expressão de sentimento positivo e Autocontrole da agressividade, respectivamente). No fator 2, há uma discrepância negativa apenas para o item 4 (Agradecer elogios), possivelmente por se tratar de demandas (elogios) pouco freqüentes na vida dessas pessoas; nos demais itens, os resultados sugerem que as mulheres obesas são bastante habilidosas e expressivas.

No fator 5 , as médias de todos os itens foram superiores ao ponto médio da escala $(2,0)$ e às da amostra de universitárias, mostrando que as mulheres obesas apresentam maior autocontrole da agressividade. O que chama a atenção é que o autocontrole pouco desenvolvido, visto na análise das HAA, parece não se generalizar para as situações de críticas e chacotas. Pode-se supor que elas tenham se dessensibilizado diante de tais situações, ou que, considerando o sobrepeso atual bastante exagerado, isso de fato já não mais ocorre.

No fator 1 (Enfrentamento e auto-afirmação com risco), as obesas têm média superior ao ponto médio do inventário (2) em apenas quatro itens (25, 17, 1 e 9); nos outros itens a média é semelhante à da amostra 
normativa ou mais baixa. No fator 3 , as médias estão mais baixas nos itens que envolvem alguma forma de assertividade $(20,28,22)$. Nos outros, conquanto inferiores às médias da amostra das universitárias (exceto no item 42), as médias encontram-se acima do ponto médio da escala. Assim, pode-se dizer que, em relação à conversação e à desenvoltura social, as mulheres obesas conseguem, com alguma freqüência, apresentar um bom desempenho social. Os resultados no fator 4 mostram que as mulheres obesas apresentam muita dificuldade com pessoas desconhecidas e com a auto-exposição, o que se torna mais crítico nos itens que demandam maior assertividade (como por exemplo, o item 26, referente a recusar pedidos).

Os valores médios (e desvios padrões) obtidos na amostra deste estudo, para cada um dos itens do HAA, aparecem na Tabela 1. Pode-se ver que, no grupo das HAA, em quatro dos seis itens, as médias foram inferiores ao ponto médio da escala $(2,0)$, ou seja, as mulheres obesas relatam muita dificuldade quando a assertividade envolve recusar alimento. Ao que parece, a dificuldade é ainda maior com conhecidos e amigos do que com desconhecidos, pelos menos nos exemplos avaliados.

Tabela 1 - Valores médios de freqüência (e desvios padrões) para cada um dos itens do HAA

\begin{tabular}{llc}
\hline Interlocutor & Itens & Média e (Desvio Padrão) \\
\hline Desconhecido & 19. Recusar novo produto alimentício no supermercado & $2,24(1,79)$ \\
\hline \multirow{3}{*}{ Conhecido } & 02. Recusar oferta de um lanche fora de hora & $1,38(1,57)$ \\
& 24. Recusar oferta para repetir o prato na refeição & $1,38(1,45)$ \\
& 40. Recuso a oferta de sobremesa & $1,48(1,57)$ \\
\hline Amigo & 29. Recusar um pedaço de doce ou salgado que o outro & $3,14(1,38)$ \\
& está comendo & $1,28(1,77)$ \\
\hline
\end{tabular}

$\mathrm{Na}$ análise da influência das características da amostra sobre os escores de habilidades sociais, não se verificou efeito significativo dos problemas de saúde $(t=-1,322 ; \mathrm{DP}=27, p=0,197)$; nem do fato de trabalharem ou não $(t=0,256 ; \mathrm{DP}=27 ; p=0,800)$ ou de tratamentos anteriores $(t=1,687 ; \mathrm{DP}=27 ; p=0,103)$. Também a escolaridade não apresentou relação com os escores do IHS $(F=0,542 ; \mathrm{DP}=5 ; p=0,743)$ e nas HAA $(F=0,145 ; \mathrm{DP}=4 ; p=0,366)$, o mesmo ocorrendo com o estado civil e escores no IHS $(F=0,711 ; \mathrm{DP}=4$; $p=0,593)$ e nas HAA $(F=0,567 ; \mathrm{DP}=4 ; p=0,689)$.

Os resultados da análise do questionário de auto-relato sobre a obesidade são apresentados descritivamente com base na freqüência de ocorrência, conforme as Tabelas 2 e 3. Como mostra a Tabela 2, a quase totalidade das respondentes relatou que deseja emagrecer por ser importante para a saúde. Este dado significa que essas mulheres já estão conscientes dos malefícios que a obesidade traz para a saúde e, por isso, demonstram preocupação com tal aspecto ou que elas também já vivem esses prejuízos e necessitam perder peso para superá-los (problemas de saúde foram relatados por setenta e dois por cento desse grupo). Também é interessante observar que essas mulheres apontaram a aceitação por outros $(51,75 \%)$ e a questão estética $(41,38 \%)$ como fatores que influenciam o desejo de perder peso. A obtenção de trabalho foi apontada como motivo por apenas $17,24 \%$. De acordo com a Tabela 3, as mulheres obesas tendem a comer mais em algumas situações específicas, principalmente as geradoras de ansiedade, de problemas e de raiva. O relato de maior consumo alimentar na ansiedade confirma a literatura médica. Por outro lado, a ansiedade parece permear as demais situações, com exceção da última (felicidade).

Tabela 2 - Freqüência de motivos relatados para a perda de peso

\begin{tabular}{lcc}
\hline Motivos & $\mathrm{N}$ & Freqüência \\
\hline Saúde & 28 & $96,55 \%$ \\
Aceitação por outros & 15 & $51,73 \%$ \\
Beleza/estética & 12 & $41,38 \%$ \\
Obtenção de trabalho & 5 & $17,24 \%$ \\
\hline
\end{tabular}


Tabela 3 - Situações em que as respondentes relataram comer em maior quantidade e/ou freqüência

\begin{tabular}{lcc}
\hline Situações & $\mathrm{N}$ & Freqüência \\
\hline Ansiedade & 24 & $82,76 \%$ \\
Problemas & 22 & $75,86 \%$ \\
Raiva de alguém & 16 & $55,17 \%$ \\
Frustração & 14 & $48,27 \%$ \\
Tristeza & 13 & $44,82 \%$ \\
Discussão & 12 & $41,38 \%$ \\
Medo & 6 & $20,69 \%$ \\
Felicidade & 1 & $3,45 \%$ \\
\hline
\end{tabular}

A comparação entre o IMC atual e o desejado é apresentada na Figura 4, que indica também os pontos de corte para peso abaixo do normal (IMC abaixo de
19), peso normal (19 a 23,9, obesidade leve (24 a 28,9), obesidade moderada $(29$ a 38,8$)$ e obesidade grave ou mórbida (acima de 39).

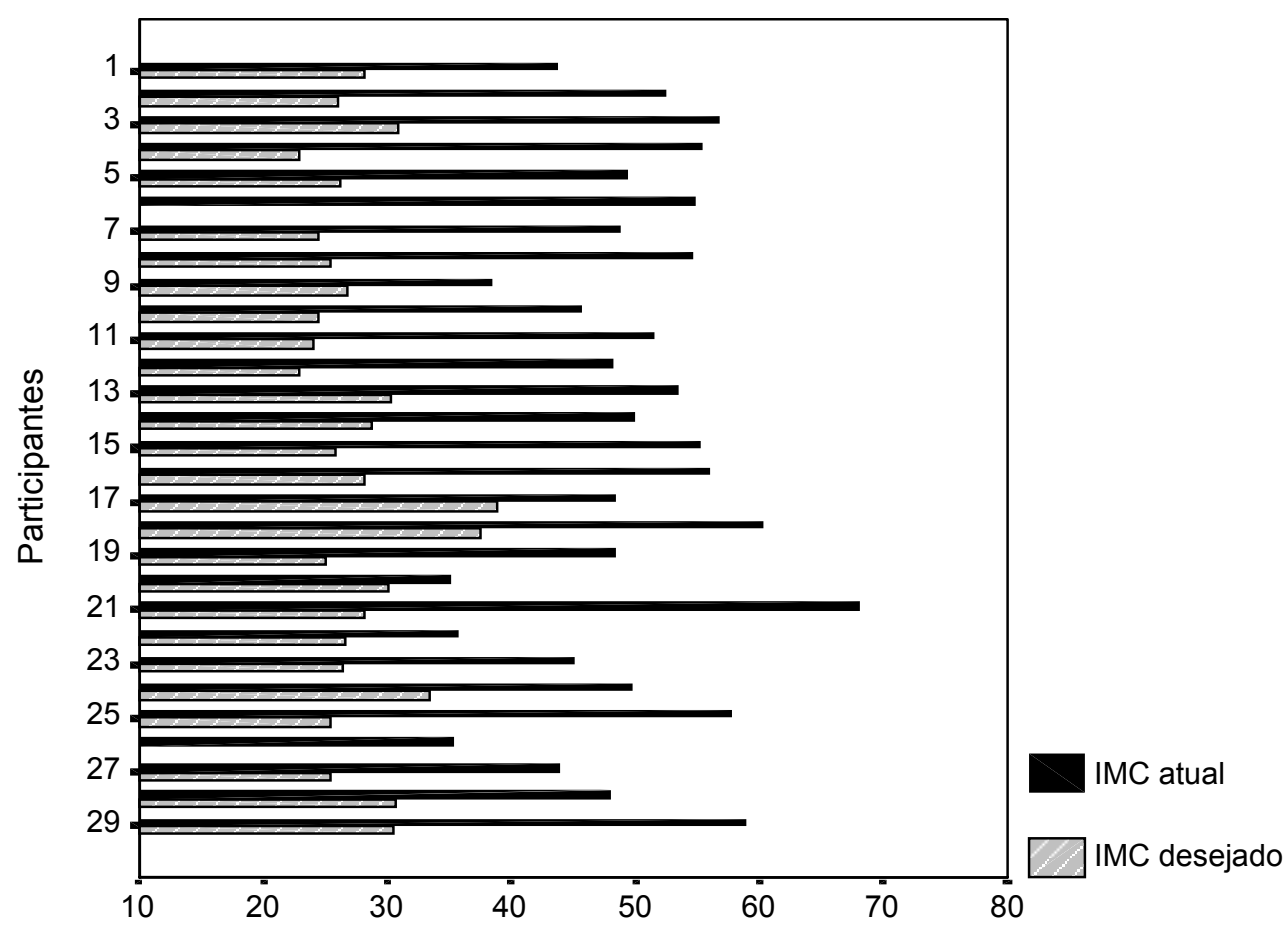

Índice de massa corpórea

Figura 4 - Comparação entre o IMC atual e o desejado pelas participantes (Obs.: As participantes 28 e 29 não mencionaram o peso que gostariam de ter)

De acordo com os dados obtidos, a média do IMC atual foi de 49,9 enquanto para a do IMC idealizado foi de 27,88 . Essa segunda média ainda se enquadra na classificação de obesidade leve, ou seja, mesmo se essas mulheres tivessem o peso que gostariam de ter, elas ainda permaneceriam obesas. Mas é interessante verificar que todas as mulheres relataram o desejo de reduzir o peso, o que significou, em geral, uma proporção acima de 100\% do IMC atual. Pela Figura 4, pode-se verificar que, se atingido o peso idealizado, apenas duas das responden- tes deixariam de ser obesas (4 e 12), enquanto que, dentre as demais, 15 ainda estariam na faixa de obesidade leve, quatro na de moderada $(3,18,25$ e 29$)$ e uma delas ainda continuaria com obesidade mórbida (17).

\section{Discussão}

Este estudo apresenta resultados que evidenciam parcialmente a hipótese inicial, ou seja, as pessoas obesas sob tratamento apresentam déficits específicos 
em habilidades assertivas relacionadas à alimentação, o que sugere uma maior dificuldade em relação a autocontrole e, conseqüentemente, de superação da doença. Os escores da habilidade social de recusar alimento foram, em média, significativamente inferiores aos de habilidades sociais gerais e, como estes foram semelhantes aos da amostra normativa, pode-se concluir que a obesidade não gera, necessariamente, dificuldades de relacionamento. Esses dados são semelhantes aos obtidos por Klesges (1984) e vão ao encontro dos de Miller e colaboradores (1995) quando afirmam que as mulheres não apresentam problemas relacionados às habilidades sociais. Todavia, contradizem o primeiro estudo de Miller e colaboradores (1990), que identificaram déficits nas habilidades sociais de mulheres obesas.

Os dados deste estudo também mostram que as mulheres obesas são mais habilidosas em alguns contextos sociais em detrimento de outros. A análise das subescalas do IHS-Del Prette mostra também que, em algumas situações, as mulheres obesas apresentam déficits maiores, como nas situações relacionadas à alimentação e na autoexposição a desconhecidos e situações novas (F4). Neste segundo caso, pode-se inferir algum receio de discriminação, possivelmente fundamentado em experiências negativas que já viveram ou que percebem como altamente provável. Isto certamente acaba dificultando que essas mulheres sejam habilidosas com estranhos. Contudo, os resultados confirmam o estudo de Miller e colaboradores (1995), em que consideraram as mulheres obesas mais habilidosas do que as outras pessoas, com melhor desempenho em habilidades de conversação e de desenvoltura social com conhecidos. Tais constatações parecem apoiar a noção, razoavelmente difundida, de que as "gordinhas são simpáticas e agradáveis", especialmente em situações de interação com pessoas conhecidas.

Um dado intrigante foi em relação às habilidades de autocontrole, nas quais as mulheres obtiveram bons resultados. Seria contraditório afirmar que as obesas apresentam habilidades de autocontrole, considerando que elas apresentam muita dificuldade no que diz respeito ao controle alimentar. Por isso, uma explicação para esses dados pode ser a de que as habilidades consideradas de autocontrole no IHS-Del Prette referem-se, especialmente, às de lidar com críticas e chacotas. Tal fato pode indicar que essas mulheres tenham desenvolvido essas habilidades em virtude dos preconceitos e até cobranças dos familiares, o que pode ser confirmado com alguns dados da análise do questionário de auto-relato da obesidade em que duas mulheres mencionaram o desejo de emagrecer para evitar a discriminação e chacotas e 44,83\% afirmaram querer emagrecer por causa de outros (familiares, cônjuge, filhos, amigos). Esses dados também estão de acordo com o caráter situacional das habilidades sociais (Del Prette \& Del Prette, 2001 e 2003), pois o autocontrole é presente em relação à agressividade, mas ausente na alimentação.

No questionário, verificou-se que situações envolvendo a ansiedade são consideradas os principais motivos das mulheres comerem em excesso, conforme relatado. Esse resultado está de acordo com os obtidos por Schachter, Goldman e Gordon (1968) que consideram o comer em excesso uma estratégia dos obesos para reduzir a ansiedade.

Os resultados não mostraram relação entre as características sociodemográficas (escolaridade, trabalhar fora de casa, problemas de saúde, estado civil) da amostra e os escores de habilidades sociais. Sendo assim, a presença de outro problema de saúde e tentativas anteriores de tratamentos para emagrecer não parecem ter influenciado os indicadores de habilidades sociais. Seria interessante, em pesquisas futuras, verificar, no entanto, se estes não seriam fatores que aparecem em grupos mais homogêneos ou quando se comparam grupos contrastantes.

Também se verificou que as mulheres obesas apresentam baixas expectativas quanto à perda de peso em relação às pessoas em geral e ao padrão de IMC saudável. Quando questionadas sobre o peso que gostariam de ter, 89\% delas ainda continuariam obesas se atingissem o peso idealizado. Uma possível explicação para esses resultados é que elas estejam ajustando suas expectativas a uma meta realista mais do que a uma meta ideal. Outra suposição é que, pelo fato de estarem discrepantes do peso considerado "saudável", as perdas idealizadas já as deixariam satisfeitas. Esse dado sugere que é importante estabelecer metas graduais no processo de tratamento desses casos, que poderiam evitar ou minimizar o abandono do tratamento.

A identificação das situações de "maior pressão alimentar" pode auxiliar os programas de tratamento de obesidade. Entretanto, o Treinamento de Habilidades Sociais em geral, e de habilidades assertivas em particular, constituiriam instrumentos auxiliares de grande ajuda na medida em que desenvolvessem habilidades alternativas para as pessoas lidarem com aquelas situações, em sua maioria, sociais.

Considerando a importância de se conhecer melhor os aspectos psicológicos envolvidos na obesidade e a influência dessa doença nas relações sociais, seriam necessários estudos futuros para verificar a relação entre obesidade e habilidades sociais em amostras maiores, inclusive para prover referência normativa que poderia auxiliar no planejamento de programas de intervenção nessa área.

\section{Referências}

ABESO - Associação Brasileira para o Estudo da Obesidade. (1997). Dados epidemiológicos sobre obesidade.

Psico-USF, v. 9, n. 2, p. 201-210, Jul./Dez. 2004 
Disponível em: http://www.abeso. org.br. Acesso em: 30/6/2004.

Almeida, S. S., Nascimento, P. C. B. D. \& Quaioti, T. C. B. (2002). Quantidade e qualidade dos produtos alimentícios anunciados na televisão brasileira. Revista de Saúde Pública, 36, 353-355.

Bandeira, M., Costa, M. Neves, Del Prette, Z. A. P., Del Prette, A. \& Gerk-Carneiro, E. (2000). Qualidades psicométricas do Inventário de Habilidades Sociais (IHS): estudo sobre a estabilidade temporal e a validade concomitante. Estudos de Psicologia, 5, 401-419.

Breiman, L., Friedman, J. H., Olshen, R. A. \& Stone, C. J. (1984). Classification and regression trees. Wasworth and Brooks Cole Advanced Books and Software: Pacific Grove, CA.

CID-10. (1998). Classificação estatística internacional de doenças e problemas relacionados à saúde. 10 rev., vol. 1 . São Paulo: EDUSP.

Conselho Federal de Psicologia. (2003). Edital CFP no 1 - Processo de avaliacão dos testes psicológicos, de 17/7/2003. Disponível em: http://www.pol.org.br. Acesso em: 10/9/2003.

Cotran, R. S., Kumar, V. \& Collins, T. (2000). Robbins: patologia estrutural e funcional. Rio de Janeiro: Guanabara Koogan.

Del Prette, A. \& Del Prette, Z. A. P. (2001). Psicologia das relações interpessoais: vivências para o trabalho em grupo. Petrópolis: Vozes.

Del Prette, A. \& Del Prette, Z. A. P. (2003). Treinamento assertivo, ontem e hoje. Em C. E. Costa, J. C. Luzia \& H. H. N. Sant'Anna (Orgs.). Primeiros passos em análise do comportamento (pp. 149-160). Santo André: ESETec.

Del Prette, G. (2002). Obesidade infantil: a influência dos comerciais de televisão sobre os hábitos alimentares (Monografia de Conclusão). São Paulo: Universidade de São Paulo.

Del Prette, Z. A. P. \& Del Prette, A. (1999). Psicologia das habilidades sociais: terapia e educação. Petrópolis: Vozes.

Del Prette, Z. A. P. \& Del Prette, A. (2001). Inventário de habilidades sociais: manual de aplicação, apuração $e$ interpretação. São Paulo: Casa do Psicólogo.

Del Prette, Z. A. P. \& Del Prette, A. (2003). Habilidades sociais e dificuldades de aprendizagem: teoria e pesquisa sob um enfoque multimodal. Em A. Del Prette \& Z. A. P. Del Prette (Orgs.). Habilidades sociais, desenvolvimento e aprendizagem: questões conceituais, avaliação e intervenção (pp. 167-206). Campinas: Alínea.
Del Prette, Z. A. P., Del Prette, A. \& Barreto, M. C. M. (1998). Análise de um Inventário de Habilidades Sociais (IHS) em uma amostra de universitários. Psicologia: Teoria e Pesquisa, Brasília, 14, 219-228.

Friedman, M. A. \& Brownell, K. D. (1995). Psychological correlates of obesity: Moving to the next research generation. Psychological Bulletin, 117, 3-20.

Guyton, A. C. \& Hall, J. E. (1996). Tratado de Fisiologia Médica. Rio de Janeiro: Guanabara Koogan.

Halpern, A. (1998). Obesidade. São Paulo: Contexto.

INAN - Instituto Nacional de Alimentação e Nutrição (BRASIL). Condições nutricionais da população brasileira: adultos e idosos. Brasília, DF: Ministério da Saúde, 1991. (Pesquisa Nacional sobre Saúde e Nutrição).

Klesges, R. C. (1984). Personality and obesity: Global versus specific measures? Behavioral Assessment, 6, 347-356.

Leite, P. F. (1996). Obesidade na clínica médica. Belo Horizonte: Health.

Miller, C. T., Rothblum, E. D., Barbour, L., Brand, P. A. \& Felicio, D. M. (1990). Social interactions of obese and non-obese women. Journal of Personality, 58 , 365-380.

Miller, C. T., Rothblum, E. D., Brand, P. A. \& Felicio, D. M. (1995). Do obese women have poorer social relationships than non-obese women? Reports by self, friends, and co-workers. Journal of Personality, 63, 65-85.

Molinari, E. \& Riva, G. (1995). Self-others perception in a clinical sample of obese women. Perceptual and Motor Skills, 80, 1283-1289.

O’Neil, P. M. \& Jarrel, M. P. (1992). Psychological aspects of obesity and dieting. Em Wadden, T. A. \& Van Itallie, T. B. (Orgs.). Treatment of seriously obese patient (pp. 252-270). New York: Guilford Press.

Schachter, S., Goldman, R. \& Gordon, A. (1968). Effects of fear, food deprivation, and obesity on eating. Journal of Personality and Social Psychology, 10(2), 91-97.

Striegel-Moore, J. \& Rodin, P. F. (1986). Toward an understanding of risk factors for bulimia. American Psychologist, 41, 246-263.

Wadden, T. A. \& Stunkard, A. J. (1985). Social and psychological consequences of obesity. Annais of Internal Medicine, 103, 1062-1067.

Recebido em agosto de 2004 Reformulado em setembro de 2004 Aprovado em novembro de 2004 
Sobre os autores:

Sabrina Kerr Bullamah Correia é psicóloga pela Universidade Federal de São Carlos.

Zilda Aparecida Pereira Del Prette é doutora em Psicologia pela USP-SP, com pós-doutorado pela Universidade da Califórnia, professora titular da UFSCar, pesquisadora do CNPq e orientadora de Pós-Graduação em Educação Especial (UFSCar) e Psicologia (USP-RP).

Almir Del Prette é doutor em Psicologia pela USP-SP, pesquisador do CNPq e orientador do Programa de PósGraduação em Educação Especial (UFSCar). 